\title{
LA POLÍTICA DEL EMPRENDIMIENTO NARANJA EN COLOMBIA, NUEVAS FORMAS DE CONTROL DE LA POBLACIÓN *
}

\section{The orange entrepreneurship policy in Colombia, new ways of population control}

Oscar Andrés López Cortés**

Víctor Manuel Moncada Prieto***

Recepción: 3 de febrero de 2020. Aceptación 30 de junio de 2020.

DOI: http://dx.doi.org/10.21017/Rev.Repub.2020.v29.a89

\section{RESUMEN}

Este artículo de investigación presenta un análisis de la política pública de emprendimiento en Colombia, la cual se ha enfocado durante los últimos años a la economía naranja. Con tal objetivo fueron analizadas leyes, documentos de política pública y mediciones de emprendimiento. Se argumenta que esta política pública no ha arrojado los resultados esperados, pero sí ha sido un elemento importante en la generación de una cultura emprendedora que se enfoca en la construcción del sujeto emprendedor. Se concluye que la cultura del emprendimiento propicia una narrativa épica que representa al emprendedor como héroe particular y paradigmático de su propia trayectoria, al tiempo que proyecta un estereotipo de sujeto hiperindividualista, ajeno a las condiciones materiales e históricas en las que vive.

* Artículo producto de investigación desarrollada en colaboración de los grupos Estudios Interdisciplinarios DESC y Mundo del Trabajo y el Grupo de Investigaciones Socio Jurídicas -GISJ del Centro de Investigaciones Socio-Jurídicas de la Universidad Libre, Sede Principal. Proyecto de Investigación: Derechos económicos, sociales y culturales de los trabajadores de la cultura en Colombia.

* Doctor en Antropología de la Universidad del Cauca. Magíster en Derecho de la Universidad Nacional de Colombia. Abogado de la Universidad Nacional. Profesor y director del Centro de Investigaciones Socio-Jurídicas de la Universidad Libre, Sede Principal. Correo electrónico: oscara.lopezc@unilibrebog.edu.co, https://orcid.org/0000-00027589-7233.

*** Abogado de la Universidad Libre. Candidato a magíster en geografía de la Universidad Nacional de Colombia. Investigador auxiliar de la Universidad Libre. Correo electrónico: ManuellMonk@gmail.com, https:/ / orcid.org/0000-0001-8809-2672. 

nuevas formas de control de la población

Palabras clave: Economía Naranja, construcción del sujeto, emprendimiento, políticas públicas.

\begin{abstract}
This paper presents an analysis about the Colombian entrepreneurship public policy, which one last years has been focusing on the orange economy. So, it has been analyzed several laws, public policy documents and measurement of entrepreneurship. It is argued that the public policy no had produced expected results, however it has been a key element in developing an entrepreneurship culture, which is focusing on building the subject. It is concluded that entrepreneurship culture encourages an epic narrative which shows the entrepreneurship like a specific hero archetype of its own trajectory, while reflects a stereotype of the subject hyper individualist who is moved away from material and historic conditions who is living.
\end{abstract}

Key words: Orange Economy, building of subject, entrepreneurship, public policies.

\title{
INTRODUCCIÓN
}

La política de emprendimiento en Colombia destinada a generar oportunidades de ingresos, principalmente para las personas más jóvenes, ha sido una de las banderas durante los últimos cinco gobiernos. Innova, Innpulsa, Proexport, Bancoldex, el Fondo de Modernización e Innovación para las Micro, Pequeñas y Medianas Empresas y Unidad de Desarrollo e Innovación son apenas algunos de los programas e instituciones creados por el Estado colombiano para canalizar recursos económicos, administrativos, informáticos, técnicos y académicos, destinados a los emprendedores. Todos estos esfuerzos realizados con capital público constituyen el elemento orgánico de la denominada Política Nacional de Emprendimiento, la cual se complementa con abundante pero dispersa legislación. Se trata de un marco institucional amplio desarrollado durante las últimas dos décadas, que ha absorbido una enorme cantidad de recursos públicos.

En los últimos años, esta política de emprendimiento ha estado cada vez más asociada a la denominada «economía naranja», estrategia mediante la cual el discurso oficial construye un universo de oportunidades infinitas de creatividad, innovación y crecimiento para los nuevos emprendedores digitales. Este tipo de políticas genera un conjunto de prácticas discursivas que, como Ludmila Costhek ha señalado, se encuentran bastante desarrolladas en el lenguaje del mercado, pero poco analizadas por las ciencias sociales. Al 
estudiar esas prácticas discursivas Costhek expone la manera como esas prácticas discursivas, como por ejemplo el e-marketplace, comprendido como universo virtual apropiado para la transformación de trabajadores en microemprendedores, se ha consolidado como un espacio hiperflexible del trabajo en el cual se han precarizado múltiples profesiones.

El caso de Colombia ilustra el argumento de Ludmila Costhek. En los últimos planes de Gobierno en este país la política de emprendimiento relacionado con la economía naranja ha adquirido cada vez mayor importancia, al punto, como se mostrará al final del artículo, de ser un pilar del más reciente plan nacional de desarrollo en Colombia. No obstante, esta política de emprendimiento deja muchos interrogantes sobre los cuales el discurso estatal ha guardado silencio. Este artículo mostrará en qué consiste la política de emprendimiento y cómo está regulada en Colombia, así como los resultados de las mediciones del emprendimiento en el país. Luego de ello se expondrán los aspectos generales de la economía naranja y la manera como esta ha adquirido un lugar central en la política de emprendimiento. Finalmente, a modo de conclusión, se presentará un análisis que busca dar respuesta al interrogante por cuál ha sido el efecto material de esta política de emprendimiento y economía naranja en Colombia.

\section{EL CONCEPTO DE EMPRENDIMIENTO}

Según María Minniti, en 1732 Richard Cantillon definió el emprendimiento como la voluntad de los individuos de desarrollar formas de intermediación que implican el riesgo económico de una nueva empresa (Minniti, 2012, p. 23). Señala la autora que dos siglos más tarde, Schumpeter definió al emprendedor como un actor económico que, arriesgando sus recursos, es capaz de convertir una nueva invención o de imitar un producto o tecnología existente para producir un cambio tecnológico comercializable y obtener un beneficio de ello.

Actualmente, la OCDE define el emprendimiento como un conjunto de prácticas que «buscan generar valor a través de la creación o expansión de actividades económicas identificando y explotando nuevos productos, procesos o mercados». El concepto ha evolucionado en años recientes hacia el de «emprendimiento dinámico», para referirse a las nuevas empresas que, pese a comenzar pequeñas, en un breve lapso temporal no solo logran sobrevivir, sino crecer significativamente hasta convertirse en PyMES o incluso en empresas de gran volumen (Kantis y Méndez, 2012 ).

Pese a que el concepto de emprendimiento dentro de la economía política apareció hace casi 3 siglos, apenas en los últimos 20 años ha adquirido la importancia que hoy tiene en las políticas públicas, las discusiones académicas y las 

nuevas formas de control de la población

estrategias diseñadas por la administración de empresas. Las ciencias sociales solo hasta hace muy poco tiempo han prestado atención al fenómeno.

Pfeilstetter (2011) revisa de manera exhaustiva las actuales nociones de emprendedor usadas en las ciencias sociales, tanto aquellas que él considera hegemónicas como las denominadas periféricas, a través de la metodología de análisis de contenido. El autor pone en contexto el concepto de emprendedor a partir de los referentes teóricos ofrecidos por autores de la sociología como Ulrich Beck, Richard Sennett, Antony Giddens, Nicklas Luhmann y Pierre Bourdieu. A partir de estas posturas, Pfeilstetter plantea que la figura del emprendedor es la expresión prototípica del sujeto liberal individualista capaz de dar respuesta al contexto liberal a través de «la construcción reflexiva, flexible y funcionalmente adecuada, de acciones racionales cuyo conjunto desemboca en una biografía única y diferenciada, capaz de enfrentar los desafíos de riesgo e incertidumbre del entorno» (p. 3). De acuerdo con esto, el autor señala que el emprendedor «es el modelo del sujeto en un mundo liberal que se está promocionando desde las instituciones... el actor tipo que mejor encaja en la economía de mercado, asumiendo su persona, en la práctica, los postulados teóricos del liberalismo» (p. 3).

Con el fin de comprender la manera como surgió el concepto de emprendedor, Pfeilstetter organiza la bibliografía de ciencias sociales sobre emprendimiento en dos categorías: teorías subjetivistas y teorías sobre el emprendedor tipo. Las primeras, presentes principalmente en la economía y en algunas corrientes de la sociología, la psicología y el derecho, son teorías que no problematizan el contexto en el cual son producidas, se fundamentan ontológicamente en el libre albedrío y metodológicamente en estudios económicos, principalmente cuantitativos y de corte longitudinal; parten de la tesis del actor racional y del individualismo que afirman elementos intrasubjetivos, como la capacidad individual, la personalidad o la configuración psicológica de los sujetos, en tanto aspectos que priman sobre el ambiente. En estas teorías, el éxito o fracaso del emprendimiento depende de decisiones racionales de los sujetos más que de aspectos propios del entorno, el cual está determinado por la existencia de otros emprendedores: «el talento de los individuos prima sobre la importancia de las redes y las acciones colectivas» (p. 5). A este primer grupo de teorías Pfeilstetter le asigna un carácter hegemónico. De otro lado y presentes en casi todas las ciencias sociales están las teorías del emprendedor tipo, las cuales parten de análisis efectuados en grupos determinados de población diferenciados por algún tipo de marcador étnico, etario, de género, por actividad económica, entre otros. Para el autor, estos estudios presentan un problema epistemológico en tanto al centrarse en grupos determinados pierden de vista los procesos de cambio social, restan importancia a la acción y la comunicación, las cuales no se rigen necesariamente por lógicas de perte- 
nencia o identificación a grupos diferenciados (p. 6). No obstante, el autor reconoce en estos estudios una bondad teórica y metodológica importante, la cual es fundamental en nuestra propia investigación: la capacidad de distinguir conceptos hegemónicos de emprendedor empleados en países ricos, de otro tipo de emprendedor vinculado de forma periférica al capitalismo.

Finalmente, el artículo propone una vuelta a los orígenes etimológicos y teóricos del término, que permite superar algunos de los problemas epistemológicos que conlleva el uso irreflexivo y reduccionista del concepto. Pfeilstetter propone operar metodológicamente con una perspectiva teórica más abstracta del emprendedor, basada en las teorías de la complejidad y a partir de allí, entender el emprendimiento "en términos de adaptación, evolución e interdependencia y ver las organizaciones creadas por emprendedores como estructuras autopoiéticas» (p. 8). Esto permitiría distanciarse de los extremos ideológicos que han caracterizado el debate sobre emprendimiento en las ciencias sociales, entre la dualidad satanización-idealización de la figura y proceder con cierta ingenuidad empírica al investigar el fenómeno.

El florecimiento del emprendimiento en las últimas dos décadas parece coincidir con la agudización de la crisis económica, la cual ha significado la pérdida masiva de empleos, la reducción de las garantías laborales, la reestructuración de las empresas y la emergencia de nuevas formas de trabajo que propenden hacia las actividades por cuenta propia y el trabajo independiente. No es casual que gobiernos locales de todo el mundo estén invirtiendo enormes cantidades de recursos económicos, físicos, técnicos y humanos, en el diseño y ejecución de políticas de emprendimiento; al mismo tiempo que organismos internacionales de diversos orígenes y propósitos, como el FMI, el BM y USAID, han promovido iniciativas para fomentar el emprendimiento, especialmente enfocadas en los países en desarrollo (Minniti, p. 23).

Uno de los aspectos en los que se invierte gran cantidad de esos recursos y en donde más intervienen Estados, entidades multilaterales y sector académico, es en los sistemas de evaluación del emprendimiento. Al respecto, existen diferentes mecanismos de medición, como el Índice de Competitividad Global (GCI, por su sigla en inglés), el ISi Prodem, el cual permite caracterizar y ordenar a los países por el nivel de desarrollo de condiciones sistémicas (sociales, económicas, legales) para el emprendimiento (Kantis y otros, 2012 , p. 8). También se encuentran el Doing Business del Banco Mundial, el Index of Economic Freedom de la Fundación Heritage, el Reporte Mundial de Competitividad del Foro Económico Mundial.

Por último, cabe destacar el Global Entrepreneurship Monitor (GEM), a través del cual se evalúa la relación entre la actividad emprendedora y el desarrollo 

nuevas formas de control de la población

económico nacional, sistema que fue creado por la Babson College y la London Business School. Son dos las particularidades de este método de evaluación que lo hacen relevante y por lo cual fue tenido en cuenta en nuestra propia investigación: el GEM fue recientemente aplicado en Colombia con la intervención de las Universidades Javeriana de Cali, Universidad del Norte, Universidad de los Andes e ICESI. La segunda es que el GEM centra su análisis desde la perspectiva del individuo emprendedor, aspecto que resulta de gran relevancia para una aproximación crítica.

Es importante destacar lo sugerido por Minniti, para quien no es fácil estandarizar los datos sobre emprendimiento, pues cada país presenta condiciones específicas que implican formas de emprendimiento distinto dependiendo de factores como el grado de desarrollo tecnológico o la disponibilidad de recursos físicos y económicos. Es por eso que en los estudios macroeconómicos es frecuente encontrar que el emprendimiento se relaciona con el autoempleo, las pequeñas empresas de nueva creación y las denominadas iniciativas start-ups (Minniti, 2012, p. 26).

En cuanto a las críticas a la política de emprendimiento, cabe destacar las que señalan la relación entre esta y el sistema educativo. Para Vicens y Grullon no se está formando adecuadamente para el emprendimiento, pues no se trata de enseñar a hacer planes de negocio, sino de fortalecer el «aprendizaje en áreas de conocimientos esenciales» (p. 6). Los autores destacan que en Estados Unidos, primer país en emprendimiento según el GCI, se ha dispuesto una red fuerte y extensa que involucra universidades, gobiernos locales y empresas, con el fin de crear espacios de educación y práctica apropiados para la generación de emprendimientos, los cuales se encuentran ligados a la innovación en ciencias y tecnología. Los programas que desarrollan algunas universidades norteamericanas, buscan generar competencias tales como «creatividad, orientación a la acción, pasión, toma de riesgos y pensamiento holístico, liderazgo, trabajo en equipo y comunicación» para promover el emprendimiento. Al respecto, Vicens \& Grullon señalan que universidades norteamericanas como Stanford, Babson y Georgia Tech, se encuentran fuertemente articuladas a los programas de emprendimiento e innovación apoyadas por el gobierno norteamericano, y se han posicionado como referentes globales en esta materia.

Otras críticas a la política de emprendimiento destacan el rol del contexto cultural, el cual puede promover o inhibir en las personas conductas más o menos emprendedoras, en tanto el surgimiento y desarrollo de nuevas empresas requiere personas con vocaciones y motivaciones específicas destinadas para ello. Por elementos del contexto cultural, los autores se refieren al «grado de valoración social del rol del emprendedor y de la innovación, la actitud frente al riesgo y la necesidad de logro de las personas, la horizontalidad y apertura de 
los empresarios para interactuar y compartir sus experiencias con terceros» (Kantis y otro, 2015, p. 6).

Minniti, en cambio, plantea su crítica a partir de aspectos geopolíticos, al afirmar que es más elevada la presencia del emprendimiento en los países más ricos y en los más pobres, siendo mucho menor el emprendimiento en los países intermedios. «En resumen, la actividad emprendedora es generalmente mayor en países con niveles más bajos de PIB que en países con rentas altas, pero se vuelve a elevar en las economías más ricas» (p. 26). Destaca la autora que aunque el emprendimiento sea más frecuente en los dos extremos de la balanza económica internacional, los efectos y las motivaciones del emprendimiento en cada polo son radicalmente distintos y la posibilidad de que nuevas iniciativas prosperen hasta convertirse en empresas exitosas, es mucho más alta en los países que disponen con mayor facilidad de desarrollo tecnológico, buena infraestructura educativa y de servicios, y eficientes sistemas de aseguramiento social. Esto se debe en parte a que en los países con mayores ingresos los emprendimientos parten de una ventaja competitiva determinada por los menores costos para acceder a mayores desarrollos tecnológicos. Las diferencias entre los países en cuanto a la relación entre emprendimiento y crecimiento económico se pueden resumir en la idea que propone Minniti como círculo virtuoso: entre mayor sea el crecimiento económico, mejores serán los resultados del emprendimiento, y a su vez, entre más emprendimiento y mejores sus resultados, mayor crecimiento económico habrá.

Estas asimetrías se reflejan en el nivel meso y micro de la economía. Minniti señala que es clara la división en la literatura entre los emprendedores que son motivados por la oportunidad y los que son motivados por la necesidad; los primeros crean una nueva empresa porque identifican una oportunidad, mientras que los segundos intentan crearla a falta de oportunidades laborales (Minniti, p. 26).

Otro dato importante que a este respecto rescata Minniti de los estudios empíricos, es la relación entre emprendimiento y desempleo, ya que son directamente proporcionales y en los países más pobres tiende a incrementarse entre las personas sin formación académica y entre las mujeres, que como en el caso colombiano, son quienes afrontan las más altas tasas de desempleo.

\section{ELMARCO NORMATIVO DEL EMPRENDIMIENTO EN COLOMBIA}

En el 2006 se expidió en Colombia la Ley 1014, conocida como Ley de Fomento a la Cultura del Emprendimiento. En ese momento la atención pública estaba 

nuevas formas de control de la población

concentrada en la reforma constitucional que daba lugar a la reelección presidencial, por lo que aquella ley pasó casi inadvertida, como muchas otras reformas tomadas en ese momento en detrimento de los derechos sociales en Colombia. La propuesta legislativa se presentaba como un mecanismo que resolvería la situación de desempleo y la falta de generación de empresa, especialmente entre la población más joven de Colombia.

La Ley 1014 definió los conceptos a los cuales se sujeta la política nacional de emprendimiento, como una

«manera de pensar y actuar orientada hacia la creación de riqueza. Es una forma de pensar, razonar y actuar centrada en las oportunidades, planteada con visión global y llevada a cabo mediante un liderazgo equilibrado y la gestión de un riesgo calculado, su resultado es la creación de valor que beneficia a la empresa, la economía y la sociedad» (Artículo 1, literal c, Ley 1014).

Los objetivos de la ley se resumen en cinco aspectos: ideológico, en tanto busca crear una cultura del emprendimiento; educativo: como fomento de un modelo de educación propicio para el emprendimiento; axiológico: al fijar los principios y valores orientadores del marco jurídico y las políticas de Estado en la materia; orgánico-funcional: marco de las herramientas e instituciones públicas promotoras de emprendimiento, como el observatorio permanente de procesos de emprendimiento y la creación de empresas como SISEA Empresa, la Red Nacional de Emprendimiento y las redes regionales; económico: expresado en la promoción de redes y articulaciones entre empresas, instituciones educativas, cadenas y clusters productivos encaminados a promover el emprendimiento.

Los frutos de la política de emprendimiento comenzaron a verse, al menos en cuanto a los objetivos centrados en la creación de un ecosistema apropiado para el emprendimiento: se creó el Fondo Emprender, administrado por el Fondo Financiero de Proyectos de Desarrollo (Fonade); se instaló además la Cátedra Virtual de Creación de Empresas, en asocio entre el Servicio Nacional de Aprendizaje (SENA) y el Ministerio de Comercio, Industria y Turismo.

Otro elemento de política pública es el Programa «emprendeT», del SENA, en virtud del cual se ofrece orientación en materia de emprendimiento a todas las personas que lo requieran, y en especial, se busca fortalecer el emprendimiento entre los aprendices adscritos a los centros de formación del SENA en carreras técnicas. El sistema de acompañamiento brindado por el SENA se divide en una primera fase de sensibilización que busca motivar a los potenciales emprendedores, seguida de la identificación de la idea de negocio encaminada a 
satisfacer una necesidad de mercado. Posterior a eso se acompaña al futuro emprendedor en la formulación de un plan de negocios que le permita identificar la mejor fuente de financiación. Por último, se brinda asesoría para la consolidación del emprendimiento.

Por su parte, la empresa privada ha promovido el programa Bogotá Emprende, el cual se ha materializado en el Centro de Emprendimiento creado a través de la alianza entre la Cámara de Comercio de Bogotá y la Alcaldía Mayor. A través de este programa se realizan encuentros, foros, capacitaciones, fortalecimiento de redes, y demás actividades destinadas a promover la cultura del emprendimiento y la creación de ideas de negocio innovadoras. También se brinda capacitación en temas claves para el éxito de un emprendimiento, como, por ejemplo: la identificación de tendencias de negocio, las habilidades que debe cultivar un buen emprendedor, la manera de potencializar el recurso humano, entre otras. Este programa es uno de los más desarrollados entre las estrategias locales de emprendimiento encargadas de las redes regionales previstas en la Ley 1014 de 2006.

Dos años después se aprobó la Ley 1258 de 2008 que creó las SAS, Sociedades por Acciones Simplificadas, que reemplazaron a las empresas unipersonales. A través de esta ley se crea una figura jurídica apropiada para las iniciativas de emprendimiento pequeñas. Sin embargo, esta Ley 1258 tuvo un efecto adicional: al amparo de promover el emprendimiento, los socios de las SAS no responden por obligaciones laborales. Señala el artículo 1 de la Ley 1258: «... el o los accionistas no serán responsables por las obligaciones laborales, tributarias o de cualquier otra naturaleza en que incurra la sociedad». Al poner en contexto la Ley 1258, es evidente que la reforma legal encubre otra forma de evasión de los derechos laborales amparándose en el objetivo de promover el emprendimiento.

En el año 2010, bajo el gobierno de Juan Manuel Santos, se expidió la Ley 1429, la cual buscaba generar condiciones adecuadas para el fomento del primer empleo y la formalización del empleo en Colombia. No obstante, el proyecto de ley original derivó en una normativa sobre renovación de la matrícula mercantil de las empresas, simplificación de trámites comerciales en materia de liquidación de empresas. Esta ley entregó beneficios tributarios a los empresarios, como exenciones y alivios en el pago de los impuestos de renta, parafiscales y otras contribuciones de nómina, en el impuesto de industria y comercio y en la matrícula mercantil. Todo lo anterior en nombre de la generación de empleo.

Dentro de las exenciones tributarias contempladas en la Ley 1429 fueron incluidas algunas destinadas a las pequeñas empresas, la cuales se define así con 

nuevas formas de control de la población

base en dos criterios: aquellas cuyo personal no sea superior a 50 trabajadores o cuyos activos no superen los 5.000 salarios mínimos legales mensuales vigentes (smlmv). Ambos criterios resultan polémicos, pues según el tipo de actividad económica una empresa de 49 trabajadores puede ser pequeña y manejar pocos recursos, o puede ser una gran empresa y percibir una enorme cantidad de ganancias. Luego el número de trabajadores no garantiza un rasero adecuado para determinar el tamaño de una empresa.

Pero es el segundo criterio el que resulta aún más cuestionable, ya no en términos de la vaguedad que promueve la ley, sino de la asignación de recursos. Según la ley, son consideradas pequeñas empresas aquellas cuyos activos no superen los $5.000 \mathrm{smlmv}$. A 2019 el salario mínimo en Colombia es $\$ 980.657$, el equivalente a US\$ 265 dólares americanos aproximadamente. Así, puede considerarse como una pequeña empresa aquella cuyos activos ronden los $\$ 4.900$ millones de pesos, o US\$1.330.000. De esa forma, el Estado colombiano contempló importantes beneficios tributarios para empresarios cuyos activos son superiores a un millón de dólares, generando así beneficios a sectores privilegiados bajo el amparo de leyes supuestamente creadas para generar empleos para las personas más jóvenes.

En el 2012, se crea iNNpulsa Colombia, como un programa de fomento del emprendimiento de alto impacto y el crecimiento extraordinario administrado por Bancoldex. A través de sus programas e iniciativas se brindan instrumentos financieros de mediano y largo plazo para empresas de rápido crecimiento o start ups. Como esta, entre otras políticas públicas enfocadas a la población joven adoptadas durante esta década, se destaca el programa presidencial Colombia Joven, regulado a través de la Ley 1622 de 2013 y el Decreto 1649 de 2014, normas mediante las cuales se creó el Sistema Nacional de Juventud «Colombia Joven», el cual es definido como «el conjunto de actores, procesos, instancias, orientaciones, herramientas jurídicas, agendas, planes, programas, y proyectos, que operativiza la ley y las políticas relacionadas con juventud» (Artículo 22, Ley 1622 de 2013). En virtud de dicho sistema, se creó la Dirección del Sistema Nacional de Juventud «Colombia Joven», dependencia adscrita al Departamento Administrativo de la Presidencia de la República. Esta dependencia fue creada mediante el Decreto 1649 de 2014 con el fin de mejorar la calidad de vida de las personas más jóvenes a través de su formación integral; el acceso a los servicios y recursos ofrecidos por los entes públicos; la participación en la creación de políticas relacionadas con el desarrollo social, político, económico, cultural, deportivo y ambiental; el acceso al trabajo, y el uso del tiempo libre.

El documento Conpes 3866 de 2016 revela que «iNNpulsa desarrolló iniciativas como: a) Héroes Fest: diseñada para promover un entorno más emprende- 
dor e innovador en Colombia con un método basado en ciencia cognitiva, b) Agencia de noticias ColombiaINN: estrategia para posicionar el tema de la innovación empresarial y el emprendimiento en las agendas nacional y regional, visibilizando casos de éxito de empresarios y emprendedores. Por su parte, Colciencias desarrolló Alianzas Regionales por la Innovación, con el fin de generar habilidades para diseñar y desarrollar proyectos de innovación a través de la cultura».

Recientemente, en julio de 2020, se presentó un nuevo proyecto de ley en materia de emprendimiento, radicado en el Senado con el número 161 de 2020. A través de esta reforma se busca fortalecer el sistema emprendedor, promover la cultura emprendedora y mejorar las condiciones de las medianas y pequeñas industrias. En esencia, el proyecto busca centralizar los recursos destinados para el emprendimiento a través de iNNpulsa y garantizar una ruta mas sencilla para que los emprendedores accedan a los recursos necesarios para la sostenibilidad de su propuesta.

Estos son apenas algunos de los programas, las iniciativas y los actores impulsados como parte de la política nacional de emprendimiento. Las cifras al respecto también son reveladoras. Entre 2006 y 2017 el Fondo Emprender asignó recursos por $\$ 451.777$ millones de pesos, cifra cercana a los 150 millones de dólares. Tan solo en el primer semestre de 2017 se habían asignado \$44.434 millones, y en julio de ese mismo año se presentó la convocatoria para la asignación de $\$ 36.000$ millones de pesos adicionales.

Innpulsa, por su parte, ha manejado el programa Capital Semilla para Emprendimientos Dinámicos Innovadores y la línea Aportes No Reembolsables para Escalamiento Empresarial a través de los cuales se destinaron durante el año 2015 8,3 y 5.4 millones de dólares, respectivamente. Innpulsa también maneja el Programa de Impulso a la Inversio?n para Empresas en Etapa Temprana, un esfuerzo orientado a apalancar recursos del sector privado y destinarlos a inversiones en empresas de reciente creación con un presupuesto cercano a los 4 millones de dólares (Kantis, 2015).

Pero ¿en qué se invierten tantos recursos? Del total de dineros destinados al emprendimiento y la innovación en Colombia se destinan mayores montos al emprendimiento y la innovación (44\%) que a la base científica que permite el desarrollo del emprendimiento $(38 \%)$ y por último al desarrollo y fortalecimiento de las capacidades institucionales (18\%). Esto significa que el dinero se le entrega a los particulares, aunque no necesariamente se tenga claro para qué o en qué se debe invertir, ya que el desarrollo científico no tiene la asignación presupuestal que permita determinar dónde debe estar el objetivo del emprendimiento (Kantis, 2015 ). 

nuevas formas de control de la población

No obstante, es difícil saber si los recursos invertidos en el marco de la política de emprendimiento realmente están produciendo la mejora de la calidad de vida de las personas a quienes se destinan, especialmente de los más jóvenes, población que, se supone, es el foco principal de esta política. Lo que sí podemos es analizar los reportes del Global Entrepreneurship Monitor (GEM) para Colombia, el cual nos dará elementos de juicio para un análisis de la política a través de los resultados de las mediciones realizadas por los mismos expertos en emprendimiento.

\section{ANÁLISIS DE LOS REPORTES GEM}

La metodología de medición del emprendimiento a nivel mundial más aceptada es la que realiza el Global Entrepreneurship Monitor (GEM). Se trata, como lo señalan Reynolds, Hayand y Camp (1999), de una red de investigación a través de la cual se hace seguimiento a la actividad emprendedora de aquellos países que voluntariamente se someten al monitoreo, cuyas bases metodológicas fueron inicialmente propuestas por el London Business School y del Babson College. Con los años, esta iniciativa de investigación se ha extendido a más de 50 países, dentro de los cuales se encuentra Colombia, que desde 2006 se ha sometido al monitoreo que se realiza a través de universidades colombianas. El producto de estos estudios será analizado en este apartado y se citarán como GEM 2016 y GEM 2017, que corresponden a los documentos que ha publicado el equipo GEM Colombia, liderado por las universidades ICESI, Pontificia Universidad Javeriana Cali y Universidad del Norte.

La tasa de actividad emprendedora, TEA por su sigla en inglés, es definida como el "porcentaje de individuos entre 18 y 64 años de edad que se identifican como empresarios nacientes o empresarios nuevos» (GEM, 2017, p. 10). Lo que evidencian los reportes del GEM para Colombia de los años 2016 y del periodo 2017-2018 es que, a pesar de la gran cantidad de recursos públicos invertidos en el emprendimiento, el comportamiento del TEA ha sido negativo. Las mediciones señalan que el porcentaje de empresas que logran superar las etapas nacientes y convertirse en empresas establecidas viene decreciendo, al pasar del 14\% en 2010 al 4,9\% en 2014. Según el informe de 2016, las principales razones del abandono empresarial están asociadas a cuestiones personales $(38,6 \%)$, baja rentabilidad $(24,4 \%)$, oportunidad de empleo $(18,8 \%)$ y problemas financieros (10,9\%). Si bien en el año 2016 las cifras mejoran, siguen indicando que solo el $8,9 \%$ de las personas que iniciaron su emprendimiento pudieron mantenerlo por más de 42 meses.

El reporte del periodo 2017-2018 es aún más desalentador. 
«La TEA de Colombia en el 2017 disminuyó de forma significativa en comparación con la del 2016, al pasar del 27,6\% al 18,9\%. Este comportamiento estuvo relacionado, en mayor medida, con la disminución de la tasa de emprendedores nacientes, al descender del $16,3 \%$ en el 2016 , al $10,8 \%$ en el 2017» (GEM, 2017, p. 22).

Y la cifra es aún más desalentadora si se tiene en cuenta el porcentaje de emprendedores que han abandonado su actividad empresarial; esto es lo que se conoce como discontinuidad empresarial. De acuerdo con las mediciones más recientes de los años 2013 a 2017 aumentó de forma constante esta cifra, así: «En el 2013 y el 2014 la tasa de discontinuos fue de 3,0\%, en el 2015 de 4,7\%, en el 2016 de 5,3\%, y en el 2017 de 4,4\%». Pese a la leve disminución que presentó en el año 2017, la tasa de discontinuidad empresarial en Colombia sigue en aumento. Desde el punto de vista metodológico advertimos una diferencia importante entre el GEM de 2017 y el del 2016. En el segundo reporte se tenía como una de las razones del abandono empresarial la oportunidad de empleo, mientras que en el último reporte esta variable no aparece. Ignoramos si no fue incluida en el instrumento de medición o simplemente ninguna de las personas encuestadas la tomó en consideración. Este dato es relevante en tanto estudios previos (López et al., 2015) muestran que el emprendimiento puede ser para la población trabajadora más joven un punto de tránsito hacia un empleo entendido como trabajo estable, formal y con garantías laborales como estabilidad y seguridad social, entre otras.

Otro problema adicional que aparece en el análisis de documentos que miden la política de emprendimiento en Colombia es la falta de seguimiento que el Estado realiza a los recursos invertidos en ella. Según el Conpes 3866, Colombia no cuenta con evaluaciones recientes del estado de las incubadoras y el entorno de apoyo al emprendimiento. El último diagnóstico de dicho apoyo fue elaborado por el Ministerio de Comercio, Industria y Turismo en el año 2011.

Pese al amplio desarrollo normativo sobre emprendimiento con el que ya cuenta Colombia, la OCDE ha señalado que hacen falta leyes tributarias que favorezcan el emprendimiento mediante tratamientos diferenciales según las distintas fases de desarrollo de las empresas. «Por ejemplo, en Colombia los emprendimientos afrontan la misma carga tributaria que las empresas consolidadas. Como resultado, Colombia se encuentra en la posición 84 entre 189 países en el indicador de apertura de empresas del informe Doing Business 2015 (Banco Mundial, 2014)» (Conpes 3866 de 2016 ).

¿Pero en qué se están invirtiendo los recursos del emprendimiento? ¿A qué se están dedicando las nuevas empresas en Colombia? Según los informes GEM 

nuevas formas de control de la población

2012, 2014 y 2016, hay un aumento progresivo de las empresas del sector terciario de servicio a consumidores; esto abarca actividades de venta al por menor, vehículos, alojamiento, restaurantes, servicios personales, salud, educación, servicios sociales y servicios de entretenimiento. Según datos del GEM de 2016, en Colombia para el año 2013 el 47,7\% de las nuevas empresas estaban en este sector, en el 2014 aumentó al 54,3\%, y en el 2016 al 67\%. Sin embargo, en el informe de 2017 se dice que el sector consumo «pasó de representar el 26,7\% del total de las empresas en el 2013 al 66,5\% en el 2017». En todo caso, y más allá de las pequeñas inconsistencias en las cifras que se presentan de un reporte a otro, ambos coinciden en que el sector consumo viene en un constante aumento. Esto indica que la economía colombiana se está moviendo hacia la comercialización de artículos importados y de venta de servicios. La afirmación se sustenta adicionalmente en la drástica disminución que ha tenido el sector de transformación, el cual disminuyó del 25,9\% que registraba en el 2013 , al 5,2\% para el 2017. Podemos entonces sostener que la política económica del país y las reformas al marco jurídico del trabajo adoptadas durante las dos últimas décadas han hecho de Colombia un país que cada vez produce menos bienes y servicios, para dedicarse a la prestación de servicios y a la comercialización de mayor cantidad de bienes importados.

En cuanto a los ingresos per cápita que genera el emprendimiento, aparece otro dato revelador: el $46,4 \%$ de los emprendedores perciben ingresos mensuales por debajo de los dos salarios mínimos (GEM, 2017, p. 40). Esto indica que casi la mitad de los emprendedores en Colombia a 2017 percibían un salario mensual que rondaba los 250 dólares mensuales; aproximadamente 8 dólares diarios.

El tipo de empresas que se generan también es indicativo: la mayoría de las empresas nuevas en Colombia son aquellas que en la legislación se designaban como unipersonales; y en segundo lugar las SAS, que reemplazaron a las unipersonales. Esto incide en la generación de empleo. Según los mismos informes citados, el promedio de empleos que generan estas nuevas empresas es muy bajo. El 70\% de las nuevas empresas en Colombia ha generado máximo 5 empleos, y el 18\% no generó ningún empleo nuevo; de manera que tan solo el $12 \%$ de las nuevas empresas generaron más de 5 empleos.

Pero ¿cuáles son las percepciones de la política de emprendimiento? La educación para el emprendimiento no está enseñando nada en materia de principios de economía de mercado, y la infraestructura comercial es inadecuada. Las políticas de gobierno son evaluadas negativamente por las personas consultadas, quienes además señalan que el apoyo financiero proviene en su mayoría de inversionistas informales. Los programas de gobierno no responden a las necesidades de los emprendedores, y la transferencia de 
investigación y desarrollo no llega a los emprendedores. Pese a todo, los reportes del GEM señalan que las personas valoran positivamente la generación de normas sociales y culturales que el gobierno promueve, y considera que esto ha generado mayor autonomía, responsabilidad y, sobre todo, esfuerzo personal de las personas que deciden crear una nueva empresa. Esto indica que las empresas parecen estar sobreviviendo más por convicción de las personas y su fe en el discurso del emprendimiento, que por las condiciones estructurales ofrecidas.

Este hecho contrasta con la aceptación sociocultural acerca de las bondades que trae la creación de nuevas empresas, la cual es cada vez más baja. Pese a que sigue siendo alta la creencia según la cual crear una empresa es una elección deseable de carrera y que esto otorga estatus y respeto a quien lo hace, va en aumento el número de personas que están cuestionando esta idea.

Pese a ello, la tasa de actividad empresarial va en aumento, lo que podría estar indicando que las personas inician un emprendimiento más por necesidad que por vocación o porque existan condiciones reales y, sobre todo, porque culturalmente sigue siendo una opción muy bien valorada.

\section{POLÍTICA PÚBLICA, ECONOMÍA NARANJA}

La bandera del emprendimiento en el actual gobierno colombiano se ha articulado con la economía naranja. Este campo es uno de los que ha captado mayor atención debido a que ha sido promocionado por el gobierno nacional e incluido en los planes de desarrollo por parte de los diseñadores de política pública. Pero ¿qué es la economía naranja y cómo se regula?

Por economía naranja el actual Gobierno de Colombia designa el campo de las industrias creativas y la cultura. En el 2017, y por iniciativa del ahora presidente de Colombia, Iván Duque, se impulsó en el Congreso de la República la aprobación de la Ley 1834, «por medio de la cual se fomenta la economía creativa ley naranja». De acuerdo con el artículo 2 de la Ley Naranja:

Las industrias creativas comprenderán los sectores que conjugan creación, producción y comercialización de bienes y servicios basados en contenidos intangibles de carácter cultural, y/o (sic.) aquellas que generen protección en el marco de los derechos de autor. Las industrias creativas comprenderán de forma genérica -pero sin limitarse a-, los sectores editoriales, audiovisuales, fonográficos, de artes visuales, de artes escénicas y espectáculos, de turismo y patrimonio cultural material e inmaterial, de educación artística y cultural, de diseño, publicidad, contenidos multimedia, software 

nuevas formas de control de la población

de contenidos y servicios audiovisuales interactivos, moda, agencias de noticias y servicios de información, y educación creativa.

Como se puede apreciar, el concepto de industrias creativas cubre un amplísimo rango de actividades que han sido conocidas como industrias culturales. A través de la Ley 1834 se creó el Consejo de Economía Naranja, dentro del cual tienen presencia únicamente instituciones gubernamentales; no tienen representación alguna los trabajadores de las denominadas industrias creativas. Esta ausencia de participación de la sociedad civil coincide con la manera como ha sido creada la política pública en esta materia.

Desde el plan nacional de desarrollo que el gobierno colombiano propuso al Congreso de la República en el 2018, el emprendimiento adquierió una importancia sin precedentes: se consagró como uno de los tres ejes estructurales junto a la legalidad y la equidad. La inversión presupuestal que el Estado colombiano hace en el emprendimiento y la economía naranja para el actual periodo es la más alta en la historia del país. De acuerdo con la Ley del Plan Nacional de Desarrollo, para el periodo 2018-2022 se invertirán más de 26 mil millones de pesos en la política naranja; esto es, en el desarrollo del emprendimiento de base artística, creativa y tecnológica para la creación de nuevas industrias. Y para la política de emprendimiento en general se destinarán 27,183 (veintisiete coma ciento ochenta y tres) billones de pesos. En total, para la política de emprendimiento y economía naranja se invertirán 27,443 (veintisiete coma cuatrocientos cuarenta y tres) billones de pesos (cifra cercana a los 9 mil millones de dólares). Este monto es superior al que se invertirá en Colombia durante este mismo periodo para ciencia, tecnología e innovación; esto es, desarrollo de sistemas nacionales y regionales de innovación integrados y eficaces y tecnología e investigación para el desarrollo productivo y social, donde se invertirán solo 21,201 (veintiuno coma doscientos un) billones de pesos. Se destinarán entonces más recursos para el emprendimiento y la economía naranja que para la investigación y el desarrollo tecnológico que se requieren para que esas inversiones sean exitosas. Podría decirse que son inversiones en un casino, donde se juega con el azar antes que con la razón, pero además con recursos que el Gobierno Nacional fácilmente destinará a particulares sin mayores controles por parte de los entes fiscales, pues de acuerdo al proyecto de ley recientemente presentado, todo se canalizará a través de iNNpulsa, que si bien depende del Ministerio de Comercio, funciona con relativa autonomía.

Por medio del Decreto 2120 de 2018, que reglamenta la Ley Naranja, se creó el Viceministerio de la Creatividad y la Economía Naranja como parte del Ministerio de Cultura. Este viceministerio está encargado de diseñar la política de economía naranja, que consagra, entre otros, mecanismos de financiación 
especial para los emprendimientos creativos. Entre dichos mecanismos se contempla crear cinco áreas de desarrollo naranja o ADN, o como las define el Decreto 2120 de 2018: «espacios geográficos delimitados y reconocidos a través de instrumentos de ordenamiento territorial o decisiones administrativas de la entidad territorial, que tengan por objeto incentivar y fortalecer las actividades culturales y creativas». Las ADN fueron incluidas dentro de la Ley del Plan Nacional de Desarrollo presentado por el Gobierno colombiano; quienes inviertan en estas zonas especiales gozarán de amplios beneficios y exenciones tributarias.

La primera que ya ha sido creada es la del Perpetuo Socorro en la ciudad de Medellín. Estas ADN tienen antecedentes en algunos modelos de emprendimientos creativos reconocidos por la literatura académica como positivos para el caso de Colombia, entre ellos el de Parque E y ParqueSoft, ambos ubicados igualmente en la ciudad de Medellín. El Parque E, o Parque de Emprendimiento en Colombia, es el resultado de la alianza entre la alcaldía de Medellín y la Universidad de Antioquia. Esta iniciativa está destinada a promover «la cultura emprendedora con herramientas en línea de diagnósticos, abiertas al público en general para que evalúen las ideas y elaboren planes de negocios, además de movilizar a su equipo de profesionales a diferentes comunidades para capacitar y asesorar a proyectos de emprendimiento» (Vicens y Grullon, 2011, p. 10). La Fundación sin ánimo de lucro Parque Tecnológico de Software, ParqueSoft, se destaca para Vicens y Grullon como un modelo de apoyo al emprendimiento. Este funciona bajo un esquema de negocio que se basa en la selección y subcontratación laboral entre los mismos emprendedores, dedicados al diseño de software. Sin embargo, Vicens y Grullon señalan que es el «consejo de directores» el que selecciona a los nuevos emprendedores, con base en el deseo que el aspirante muestre por «invertir su energía, pasión y talentos, en tomar riesgos y trabajar arduamente para alcanzar sus metas» (p. 10), antes que por el plan de negocio o la hoja de vida que cada uno presente.

\section{DISCUSIÓN}

La propuesta de Pfeilstteter para el análisis del emprendimiento, según la cual la metodología de investigación apropiada para las ciencias sociales debe partir de una abstracción de la figura del emprendedor para observarlo con un carácter autopoiético, en nuestra perspectiva, resulta inviable. El caso de Colombia evidencia que el emprendimiento se encuentra profundamente enraizado en las políticas públicas nacionales y, al mismo tiempo, fuertemente articulado a dinámicas globales como las del e-marketplace, a través de las cuales se fomenta la creación de nuevas empresas denominadas startups, en las que el 

nuevas formas de control de la población

lucro obtenido del trabajo humano aumenta desproporcionadamente las ganancias de las grandes empresas de tecnología y empobrece a los trabajadores del mundo.

Observar esto en abstracto es pretender ignorar la compleja red de relaciones que se tejen entre la geografía política del nuevo capitalismo, concentrado en la absorción de valor a través de las tecnologías digitales de la información y las comunicaciones, y las políticas nacionales dispuestas desde los organismos internacionales de crédito, a través de las cuales se crean subjetividades laborales. Este tipo de subjetividad creado por la política del emprendimiento naranja es transversal y, de alguna manera, indiferente a las distinciones de género, raza, nivel de educación, nacionalidad, ideología, entre otras, y al mismo tiempo, puede valerse de esas diferencias para aumentar el rendimiento de las grandes empresas del mundo. Es necesario investigar críticamente desde las prácticas concretas de gobierno de las poblaciones que tienen lugar en el espacio donde se entrelazan las políticas nacionales y las agendas globales del discurso del desarrollo.

Entre los discursos que fomentan las políticas del desarrollo asociadas al emprendimiento se encuentra el mecanismo de medición conocido como Global Entrepreneurship Monitor, iniciativa liderada a nivel mundial por Babson College y London Business School, y a nivel local, por las universidades ICESI, Javeriana de Cali y Uninorte, así como la Universidad de Los Andes, que en el 2006 hizo parte del equipo GEM en Colombia. Unas y otras universidades son consideradas de elite. En el caso local, son las instituciones de educación superior que son reconocidas como nicho de empresarios. El origen de esas universidades puede marcar una tendencia a medir el emprendimiento con los parámetros producto de los sesgos ideológicos propios de quienes financian, investigan, enseñan y se educan en esas universidades. Y a nivel global, el rasero de éxito alcanzado por estas universidades en materia de emprendimiento es el que marca el estándar de medición local. No pretendemos con ello descalificar ni el método ni los resultados de la medición. Simplemente, es necesario recordar la relación entre saber, poder y subjetividad tan analizada en el trabajo de Michel Foucault. No creemos en la existencia de los saberes independientes, neutrales o apolíticos; al contrario, partimos de una hipótesis que relaciona el saber con el poder en la construcción de las subjetividades. Como lo planteó Foucault, formaciones, saber, relaciones de poder y mecanismos de constitución del sujeto se entrelazan para formar dispositivos a través de los cuales es posible orientar la conducta de las personas. A través de lo que Foucault denominó como el dispositivo saberpoder, es posible el «acoplamiento serie de prácticas-régimen de verdad mediante el cual es posible hacer que algo inexistente se convierta en algo real, esto es, lo que se dice sobre el sujeto pueda ser sometido de forma legítima a la división verdadero/falso» (Foucault, 2007, p. 37). 
Como resultado de las mediciones realizadas por el GEM para Colombia, es fácil advertir que la política del emprendimiento no está cumpliendo los objetivos formalmente previstos de inclusión, sostenibilidad y bienestar, especialmente entre la población más joven. Aunque las normas legales analizadas hayan dado prioridad a la promoción de una «cultura emprendedora», postergando incluso las medidas técnicas y administrativas concretas que permitan la consolidación de un ambiente adecuado para la sostenibilidad de los emprendimientos, es notoria la manera como las mediciones argumentan que la cultura del emprendimiento tiene cada vez mayor acogida en el contexto colombiano.

Sin embargo, la política de emprendimiento no está generando un número importante de empleos. Por el contrario, está promoviendo la generación de empresas compuestas por una sola persona o por muy pocas. Además, está promoviendo la generación de empresas dedicadas al sector de comercialización y servicios, abandonando así la necesidad de generar empresas que produzcan nuevos bienes, haciendo que nuestra economía sea cada vez más dependiente de las importaciones. A esto se debe sumar el hecho de que la política de emprendimiento está generando ingresos per cápita de muy bajo nivel, lo que aumenta las dificultades económicas de amplios sectores poblacionales, especialmente de los más jóvenes.

En el caso de Colombia, el cierre de empresas o la incapacidad de sostener las creadas recientemente, medido como abandono empresarial, así como la falta de seguimiento y evaluación de la política de emprendimiento, denota la incapacidad de la política pública de acompañar a largo plazo a los emprendedores, así como el descuido de la base científica que permita entender en qué y para qué se deben invertir los recursos, en qué y para qué se deberían crear nuevas empresas. Desde el punto de vista estructural, esto quedó en evidencia al observar la cantidad de dinero que invierte el Estado en la política de emprendimiento, incluyendo la economía naranja, en comparación con los recursos que invierte para el avance de la investigación, la ciencia y la innovación tecnológica.

El escenario descrito demuestra lo que en otro momento, y a partir del concepto de uberización del trabajo, ha planteado Ludmila Costhek: los nuevos procesos de flexibilización laboral, entre los cuales podemos contar con la iniciativa del emprendimiento naranja impulsada por el gobierno colombiano, transfieren a las personas que trabajan en las industrias culturales la administración, los costos y los riesgos asociados a la producción de servicios y bienes inmateriales, sin que ello signifique la pérdida de control de la producción, que permanece en manos del capital. 
En el caso de la economía naranja, la política de emprendimiento se vale de dos aspectos propios de la cultura contemporánea: el uso intensivo de tecnologías digitales de la comunicación y la información por parte de las nuevas generaciones de trabajadores, la manera como estas generaciones convierten dichas tecnologías en parte de su vida cotidiana en tanto herramienta de trabajo y de consumo a la vez, y por otro lado, la conjugación de altos índices de desempleo que afectan con mayor fuerza a las personas más jóvenes, la pérdida de credibilidad que ha experimentado la educación formal entre este mismo sector generacional (como muestra el descenso de las matrículas en las universidades colombianas), y la naturalización de un entorno laboral precario como el único posible. En un escenario semejante es muy fácil convencer a las personas más jóvenes de que la mejor oportunidad laboral que tienen es la del emprendimiento, más aún si esta se encuentra asociada al uso de tecnologías digitales de la información y las comunicaciones, que se asumen como una ventaja que este grupo etario tiene. Sin embargo, no es cierto que los jóvenes «vengan con el chip» de la tecnología; como si las personas más jóvenes estuvieran por definición mejor adaptados a la tecnología. El hecho de que ellos pasen más tiempo en Facebook, Twitter o Instagram, o que satisfagan sus necesidades más habitualmente a través de aplicaciones como Rapi o Uber, no necesariamente los hace más hábiles para entender el algoritmo que hace eso posible, o para desencriptar los códigos de programación de las aplicaciones que usan a diario. Eso es lo que diferencia al usuario final de un programa, un producto de software o una aplicación, de aquel que diseña y realmente entiende cómo funcionan estas tecnologías y, por ende, quien será el sujeto empleable o el emprendedor de la economía naranja, como la promueve el gobierno colombiano.

Para culminar, es posible entonces afirmar que hay una cultura global del emprendimiento caracterizada por una narrativa épica que representa al emprendedor como héroe particular y paradigmático de su propia trayectoria, al tiempo que proyecta un estereotipo de sujeto hiperindividualista, ajeno a las condiciones materiales e históricas en las que vive; esta cultura, por una lado, reproduce una subjetividad laboral insolidaria, fragmentada, dependiente del capital y del consumo; y por otro, legitima la transferencia directa de recursos públicos a particulares, modelo que es presentado como inversión social, lo que distrae el desinterés estatal en la generación de empleos y formas dignas de trabajo.

Como afirma Arturo Escobar, el aparato del desarrollo está en capacidad de crear de manera sistemática categorías de clientes, grupos poblacionales hacia los cuales se enfocan los programas del desarrollo con el fin de sacarlos del atraso, entre los cuales Escobar señala los malnutridos, los pequeños agricultores, los agricultores sin tierra, las mujeres lactantes, entre otros (Escobar, 1998). Cabe preguntarse si los jóvenes podrían ser considerados otra sección 
dentro de la categoría de clientes a los cuales se enfocan contemporáneamente los programas del desarrollo que, como Escobar señala, se encuentran asociados a procesos económicos, políticos, culturales y discursivos.

\section{REFERENCIAS}

Costhek, L. (2018). Uberización: nuevas formas de control, organización y gestión del trabajo. En Trabajo, Derecho y Subjetividad, 25-4. O. López y A. Cuevas. Ed., Bogotá: Universidad Libre.

Conpes 3866 (2016). Departamento Nacional de Planeación. Consejo Nacional de Política Económica y Social. República de Colombia. 3866 política Nacional de Desarrollo Productivo. Recuperado en: https:/ / colaboracion.dnp.gov.co/CDT/ Conpes/Económicos/3866.pdf

Escobar, A. (1998). La invención del tercer mundo. Construcción y Deconstrucción del Desarrollo. Bogotá: Editorial Norma.

Foucault, M. (2007). Nacimiento de la biopolítica: Curso en el Collège de France: 1978-1979. Buenos Aires: Fondo de Cultura Económica.

GEM. Global Entrepreneurship Monitor Colombia. (2016). Actividad Empresarial Colombiana, 1-100.

GEM. Global Entrepreneurship Monitor Colombia. (2017). Estudio de la Actividad Empresarial 2017, 1-71, Bogotá.

Kantis, H. Federico, J. Magendzo, A. (2015). Condiciones sistémicas e institucionalidad para el emprendimiento y la innovación. Hacia una agenda de integración de los ecosistemas en los países de la Alianza del Pacífico. Banco Interamericano de Desarrollo, noviembre de 2016. Disponible en: https://publications.iadb.org/ publications/spanish/document/Condiciones-sistémicas-e-institucionalidadpara-el-emprendimiento-y-la-innovación-Hacia-una-agenda-de-integración-delos-ecosistemas-en-los-pa\%C3\% ADses-de-la-Alianza-del-Pac\%C3\%ADfico.pdf

López, O. A., Vargas, L., Covelli, C., Pérez, M. A., \& Santamaría, L. P. (2015). Sindicalismo y trabajo: la experiencia de los jóvenes bogotanos desde sus repertorios interpreta- tivos. Universitas Psychologica, 14(5), 1795-1808. Doi: https://doi.org/ 10.11144/Javeriana.upsy14-5.stej

Minniti, M. (2012). Economía Industrial. Revista. 383 (Ejemplar dedicado a: Mujer y desafío emprendedor en España), 23-30, Publicación del Ministerio de Industria, Energía y Turismo de España. 

nuevas formas de control de la población

Pfeilstetter, R. (2011). El emprendedor. Una reflexión crítica sobre usos y significados actuales de un concepto. Gazeta de Antropología, 27(1), 1-11.

Reynolds, M. Hay, R.M. Camp. (1999). Global Entrepreneurship Monitor. Executive report London School Business. Babson College, Kauffman Center for Entrepreneurial Leadership, London.

Vicens, L. \& Grullón, S. (2011). Innovación y emprendimiento: Un modelo basado en el desarrollo del emprendedor. En $V$ Foro de Competitividad de las Américas para el Banco Interamericano de Desarrollo y el Compete Caribbean, República Dominicana. 